\title{
Update on chemoprevention in BRCA1 and BRCA2 mutation carriers
}

\author{
M. Stumacher, S. M. Domchek \\ Department of Medicine, Abramson Cancer Center, University of Pennsylvania, Philadelphia, PA, USA.
}

\begin{abstract}
Chemoprevention with tamoxifen and oophorectomy are thought to be effective in decreasing the incidence of breast cancer in women at increased risk for the disease. There is mounting data supporting the idea that hormonal interventions that reduce estrogen exposure to breast epithelium, such as prophylactic oophorectomy and tamoxifen, are effective in breast cancer prevention in both BRCA1 and BRCA2 mutations carriers. Several recent studies directly address the protective effect of tamoxifen and oophorectomy in BRCA mutation carriers and suggest that these endocrine manipulations decrease the risk of primary and secondary breast cancers. Ongoing studies aim to better define the effect of tamoxifen in these very high-risk women and determining whether factors, such as earlier age of use or prior prophylactic oophorectomy, impact tamoxifen's effect. Based on existing data, we recommend that women with deleterious mutations in BRCA1 or BRCA2 be informed of the beneficial effect of oophorectomy on breast cancer risk and that women who choose breast cancer screening instead of prophylactic mastectomy be offered tamoxifen as a prevention option.
\end{abstract}

Keywords: BRCA1; BRCA2; Chemoprevention; Mutation carriers; Oophorectomy; Tamoxifen

\section{Introduction}

The Breast Cancer Prevention Trial (BCPT) conducted by the National Surgical Adjuvant Breast and Bowel Project (NSAPB) involved more than 13000 women and demonstrated a $49 \%$ reduction in invasive breast cancers in women at high risk for the disease who took tamoxifen for 5 years [1]. While tamoxifen decreased the incidence of estrogen receptor (ER) positive cancers by $69 \%$, there was no apparent effect on ER negative cancers. However, whether this effect is that of prevention or that of treatment of subclinical ER positive breast cancer is not clear. Women enrolled in

Correspondence to: Susan M. Domchek, MD, Ann B. Young Assistant Professor in Cancer Research, Department of Medicine, Abramson Cancer Center, University of Pennsylvania, 14 Penn Tower, Philadelphia, PA 19104, USA. E-mail: susan.domchek@uphs.upenn.edu; Tel: +1 215615 3360; Fax: +12156626060

Received 02/05/05

Accepted 16/06/05

First published online 30/09/05

BCO/289/2004/FO
BCPT who had first degree relatives with breast cancer benefited as much in taking tamoxifen as those without a family history. Although interim results published from two other studies have not yet demonstrated a benefit of tamoxifen [2,3], a third - the International Breast Cancer Intervention Study (IBIS-1) - demonstrated a $32 \%$ reduction in breast cancer in the tamoxifen arm [4]. These trials raise the hope that tamoxifen and possibly other hormonal interventions may be beneficial in very high-risk women, such as those with BRCA1 and BRCA2 mutations who are estimated to have a lifetime risk of breast cancer of between $60 \%$ and $80 \%[5,6]$ and a 10 -year risk of contralateral breast cancer up to $40 \%$ [7].

\section{Estrogen receptor status in BRCA-related and sporadic tumors}

Approximately $70-80 \%$ of BRCA1-associated breast cancers are ER negative [8-14], compared to $30 \%$ of sporadic tumors [15] and BRCA2-associated tumors [12-14,16]. Women under the age of 50 more 
frequently have ER negative breast cancer, both in the setting of sporadic disease and BRCA1 mutations. Moreover, the median age of onset of BRCA1-related breast cancers is significantly younger than in sporadic disease and in BRCA2-related tumors. In a small study, breast cancers in women over the age of 50 were equally likely to be ER positive in sporadic disease and BRCA1-related disease. Women under the age of 50 were more often ER negative, but the effect was more dramatic in BRCA1 positive women [17]. The implication of age of onset of disease and ER status, as well as the effect of early tamoxifen use in BRCA1 and BRCA2 mutation carriers, remains unclear. Arguments have been made that if tamoxifen preferentially reduces ER positive breast cancers, there will be little effect in predominantly ER negative BRCA1-related breast cancers. However, this assumes that BRCA1 positive women are not impacted by hormonal intervention.

The presumption that women with BRCA1 mutations are unresponsive to hormonal manipulations for prevention is not supported by data regarding prophylactic oophorectomy. In addition to decreasing the risk of ovarian cancer by $95 \%$ in BRCA1 and BRCA2 mutation carriers, prophylactic oophorectomy also decreases the risk of breast cancer with a risk ratio (RR) of 0.47 (95\% confidence interval (Cl) 0.29-0.77) [18-20]. Women undergoing oophorectomy prior to age 35 have a $61 \%$ decrease in the risk of breast cancer [19]. Therefore, one should not assume that a preponderance of ER negative breast tumors in BRCA1 mutation carriers means that the development of these tumors cannot be altered by changes in estrogen exposure.

\section{Contralateral breast cancer}

Data from the Hereditary Breast Cancer Clinical Study Group provide evidence that hormonal strategies can reduce the substantial risk of contralateral breast cancers known to exist in high-risk women. In a case-controlled study, Narod et al. [21] examined 593 women with BRCA1 or BRCA2 mutations: 209 cases with bilateral breast cancer and 384 controls with unilateral breast cancer matched for year at birth, age at diagnosis of initial breast cancer, mutation type, and residence. Adjuvant tamoxifen use following initial breast cancer diagnosis, oophorectomy, parity, smoking, radiotherapy, and chemotherapy were all examined. Tamoxifen use, adjuvant chemotherapy use, and oophorectomy were all significantly more frequent in controls than in cases (all with $P$ values $<0.004$ ), evidence that these interventions decreased the incidence of contralateral breast cancer. Tamoxifen use was reported for $13 \%$ of BRCA1 positive and $33 \%$ of BRCA2 positive women, but unfortunately ER status was available for few of the primary tumors and therefore the rationale for the use of tamoxifen in these patients was not explicit. The protective effect of tamoxifen was evident for the BRCA1 subgroup (RR $0.38,95 \% \mathrm{Cl} 0.19-0.74)$ but not the BRCA2 subgroup (RR $0.63,95 \% \mathrm{Cl} 0.20-1.5$ ), possibly due to a smaller sample size in the latter group. Oophorectomy was protective for contralateral breast cancers, particularly in women under 50 for whom the RR was 0.31 (95\% Cl 0.15-0.67). On multivariate analysis, oophorectomy, chemotherapy use, and tamoxifen use (odds ratio (OR) $0.50, \mathrm{Cl} 0.28-0.89$ ) were all independently protective of contralateral breast cancer [21].

Metcalfe and colleagues [7] collected data on 491 women with stage I or II breast cancer who were diagnosed before age 65 and had a familial BRCA1 or BRCA2 mutation to generate estimates of the risk of contralateral breast cancer and to identify host and treatment-related factors that modify the risk. The overall actuarial risk of contralateral breast cancer was $29.5 \%$ at 10 years. In this cohort, $43 \%$ of women had undergone oophorectomy and $30 \%$ had taken tamoxifen. Among the factors predictive of reduced risk of contralateral breast cancer were use of tamoxifen (hazard ratio (HR) $0.59,95 \% \mathrm{Cl} 0.35-1.01, P=0.05$ ) and oophorectomy (HR $0.44,95 \% \mathrm{Cl} 0.21-0.91$, $P=0.03$ ). The beneficial effect of oophorectomy was greater in women diagnosed prior to age 50 (HR 0.24, 95\% Cl 0.07-0.77, $P=0.02$ ). Indeed, for women diagnosed before age 50 years, the combination of tamoxifen and oophorectomy led to an HR of $0.09(95 \% \mathrm{Cl} 0.01-0.68, P=0.02)$. The 10-year risk of contralateral breast cancer in BRCA1 carriers with hormonal interventions (oophorectomy or tamoxifen) was $18.8 \%$ vs. $43.4 \%$ in BRCA1 carriers without such interventions. For BRCA2 carriers, the 10 -year risks were $13.1 \%$ vs. $34.6 \%$, respectively.

At the San Antonio Breast Symposium 2003, Pierce et al. [22] presented data from a separate study that also demonstrated the role of oophorectomy in decreasing contralateral breast cancer.

\section{Breast cancer prevention}

The ability of tamoxifen to decrease contralateral breast cancer in BRCA1 and BRCA2 mutation carriers may predict benefit in the prevention setting, as it has for women with more broadly defined risk of breast cancer. The NSAPB BCPT demonstrated the ability of tamoxifen to significantly decrease the incidence of breast cancer in women at increased risk defined as (1) age 60 years or older, (2) 5-year predicted Gail risk of at least $1.66 \%$, or (3) a history of lobular carcinoma in situ. Tamoxifen reduced the risk of invasive breast cancer by $49 \%$ and noninvasive breast cancer by $50 \%$ [11]. The only prospective data regarding 
the effect of tamoxifen on breast cancer risk in BRCA1 and BRCA2 mutation carriers come from the BCPT. King et al. performed full sequencing for BRCA1 and BRCA2 on samples from 288 of the 320 women who developed breast cancer after entering the BCPT [23]. For 32 women, DNA was either not available or could not be obtained from the stored buffy coat. Deleterious mutations in BRCA1 or BRCA2 were found in 19 women (6.6\%). Given the randomized study design, equal number of mutation carriers should have been assigned to either the tamoxifen or placebo group. Of eight women with BRCA1 mutations, five received tamoxifen, three did not, with an RR of 1.67 (95\% Cl 0.32-10.70). Of 11 women with BRCA2 mutations, three received tamoxifen, eight did not (RR 0.38, 95\% Cl 0.06-1.56). Although this would correspond to a $62 \%$ reduction in breast cancer incidence in women with BRCA2 mutations, the Cls in both groups are wide (particularly for BRCA1) and cross 1.0 [23]. Therefore, due to the very small numbers of patients in each group the results are not statistically significant and should not be used to imply that tamoxifen will not be useful for prevention in BRCA1 and BRCA2 mutation carriers, particularly when data in secondary prevention suggest otherwise.

Data also exists for the role of oophorectomy in reducing the development of primary breast cancer in BRCA mutation carriers. In a multicenter, casecontrol study, Rebbeck et al. [19] determined the incidence of breast cancer in 241 BRCA mutation carriers of whom 99 had undergone prophylactic oophorectomy and 142 were matched controls. None had previous breast cancer or mastectomy. Mean follow-up was 8.8 years. The risk of breast cancer was significantly reduced in the group undergoing oophorectomy (HR 0.47, 95\% Cl 0.29-0.77). The protective effect appeared strongest among women younger than 35 years at the time of oophorectomy (HR 0.39, 95\% Cl 0.15-1.04). Prophylactic oophorectomy also had the advantage of preventing ovarian cancer in the 551 BRCA mutation carriers followed in this same study (HR 0.04, 95\% Cl 0.01-0.16) [19].

\section{Decision analyses}

Several decision analyses support the role of tamoxifen for prevention in women with BRCA1 and BRCA2 mutations [24-26]. Duffy and Nixon [24] estimated the reduction in risk of breast cancer with the administration of tamoxifen by incorporating both prevention and treatment trials and modeling the effect on ER positive and negative tumors. The estimated breast cancer risk reduction was $13 \%$ in BRCA1 positive women (RR 0.87, 95\% $\mathrm{Cl} 0.68-1.11)$ and $27 \%$ in BRCA2 positive women (RR $0.73,95 \% \mathrm{Cl}$
0.59-0.90). Schrag et al. [25] performed decision analysis using the Markov model and found that a 30-year-old female with early stage breast cancer and a BRCA mutation gains $0.4-1.3$ years in life expectancy from tamoxifen therapy and 0.2-1.8 years from prophylactic oophorectomy. By Markov modeling of outcomes, Grann et al. [26] found that a 30 -year-old woman could prolong her survival by 1.8 years with tamoxifen and 4.6 years with both tamoxifen and prophylactic oophorectomy, incorporating estimates of tamoxifen's benefits from the data of Narod et al. Interestingly, quality-adjusted survival was found to be greatest with tamoxifen and oophorectomy (6.3 years) as compared to both mastectomy and oophorectomy (2.6 years) [26]. Therefore, even if tamoxifen only has a modest benefit, it may be preferable over mastectomy to some women from a quality of life perspective.

\section{Conclusions}

While uncertainty remains on the benefit of tamoxifen in breast cancer risk reduction in BRCA1 and BRCA2 mutations carriers, data are accumulating that suggest that interventions that reduce estrogen exposure to breast epithelium by any mechanism will reduce breast cancer in this high-risk group of women. Further work needs to be done to determine whether factors, such as earlier age of use or prior prophylactic oophorectomy impact tamoxifen's effect. Until further data are available regarding these issues and the use of other potential chemopreventive agents (such as raloxifene, aromatase inhibitors, and COX2 inhibitors), BRCA1 and BRCA2 carriers should be offered prophylactic oophorectomy both to reduce ovarian cancer and breast cancer risk. If a woman has chosen breast cancer screening instead of prophylactic mastectomy, she should be offered tamoxifen or chemoprevention study options until further information is available.

\section{References}

1. Fisher B, Costantino JP, Wickerham DL, et al. Tamoxifen for prevention of breast cancer: report of the National Surgical Adjuvant Breast and Bowel Project P-1 Study. J Natl Cancer Inst 1998; 90: 1371-1388.

2. Powles T, Eeles R, Ashley S, et al. Interim analysis of the incidence of breast cancer in the Royal Marsden Hospital tamoxifen randomized chemoprevention trial. Lancet 1998; 352: 98-101.

3. Veronesi U, Maisonneuve P, Costa A, et al. Prevention of breast cancer with tamoxifen: preliminary findings from the Italian randomized trial among hysterectomised women. Italian Tamoxifen Prevention Study. Lancet 1998; 352: 93-97.

4. Cuzick J, Forbes J, Edwards R, Baum M, et al. IBIS investigators. First results from the International Breast 
Cancer Intervention Study (IBIS-I): a randomized prevention trial. Lancet 2002; 360: 817-824.

5. Brose MS, Rebbeck TR, Calzone KA, Stopfer JE, Nathanson KL, Weber BL. Cancer risk estimates for BRCA1 mutation carriers identified in a risk evaluation program. J Natl Cancer Inst 2002; 94: 1365-1372.

6. Thompson D, Easton DF. Cancer Incidence in BRCA1 mutation carriers. J Nat/ Cancer Inst 2002; 94: 1358-1365.

7. Metcalfe K, Lynch HT, Ghadirian P, et al. Contralateral breast cancer in BRCA1 and BRCA2 mutation carriers. $J$ Clin Oncol 2004; 22: 2328-2335.

8. Verhoog LC, Brekelmans CT, Seynaeve C, et al. Survival and tumour characteristics of breast-cancer patients with germline mutations of BRCA1. Lancet 1998; 351: 1316-1321.

9. Robson M, Gilewski T, Haas B, et al. BRCA-associated breast cancer in young women. J Clin Oncol 1998; 16: 1642-1649.

10. Phillips KA, Nichol K, Ozcelik $\mathrm{H}$, et al. Frequency of p53 mutations in breast carcinomas from Ashkenazi Jewish carriers of BRCA1 mutations. J Natl Cancer Inst 1999; 91: 469-473.

11. Quenneville LA, Phillips KA, Ozcelik H, et al. HER-2/neu status and tumor morphology of invasive breast carcinomas in Ashkenazi women with known BRCA1 mutation status in the Ontario Familial Breast Cancer Registry. Cancer 2002; 95: 2068-2075.

12. Karp SE, Tonin PN, Begin LR, et al. Influence of BRCA1 mutations on nuclear grade and estrogen receptor status of breast carcinoma in Ashkenazi Jewish women. Cancer 1997; 80: 435-441.

13. Loman N, Johannsson O, Bendahl PO, Borg Å, Ferno M, Olsson $\mathrm{H}$. Steroid receptors in hereditary breast carcinomas associated with BRCA1 or BRCA2 mutations or unknown susceptibility genes. Cancer 1998; 83: 310-319.

14. Lynch BJ, Holden JA, Buys SS, Neuhausen SL, Gaffney DK. Pathobiologic characteristics of hereditary breast cancer. Hum Pathol 1998; 29: 1140-1144.

15. Early Breast Cancer Trialists' Collaborative Group. Tamoxifen for early breast cancer: an overview of the randomized trials. Lancet 1998; 351: 1451-1467.

16. Agnarsson BA, Jonasson JG, Bjornsdottir IB, et al. Inherited BRCA2 mutation associated with high-grade breast cancer. Breast Cancer Res Treat 1998; 47: 121-127.
17. Vaziri SA, Krumroy LM, Elson P, et al. Breast tumor immunophenotype of BRCA1-mutation carriers is influenced by age at diagnosis. Clin Cancer Res 2001; 7: 1937-1945.

18. Rebbeck TR, Levin AM, Eisen A, et al. Breast cancer risk after bilateral prophylactic oophorectomy in BRCA1 mutation carriers. J Natl Cancer Inst 1999; 91: 1475-1479.

19. Rebbeck TR, Lynch HT, Neuhausen SL, et al. Prophylactic oophorectomy in carriers of BRCA1 or BRCA2 mutations. New Engl J Med 2002; 346: 1616-1622.

20. Kauff ND, Satagopan JM, Robson ME, et al. Risk-reducing salpingo-oophorectomy in women with a BRCA1 or BRCA2 mutation. New Engl J Med 2002; 346: 1609-1615.

21. Narod SA, Brunet JS, Ghadirian P, et al. Tamoxifen and risk of contralateral breast cancer in BRCA1 and BRCA2 mutation carriers: a case-control study. Hereditary Breast Cancer Clinical Study Group. Lancet 2000; 356: 1876-1881.

22. Pierce LJ, Levin AM, Rebbeck T, et al. Ten-year outcomes of breast-conserving surgery (BCS) and radiotherapy $(\mathrm{RT})$ in women with breast cancer $(\mathrm{BC})$ and germline BRCA1/2 mutations: results of a multi-institutional collaboration. Breast Cancer Res Treat 2003; 82: S7.

23. King MC, Wieand S, Hale K, et al. Tamoxifen and breast cancer incidence among women with inherited mutations in BRCA1 and BRCA2: National Surgical Adjuvant Breast and Bowel Project (NSABP-P1) Breast Cancer Prevention Trial. JAMA 2001; 286: 2251-2256.

24. Duffy SW, Nixon RM. Estimates of the likely prophylactic effect of tamoxifen in women with high-risk BRCA1 and BRCA2 mutations. Br J Cancer 2002; 86: 218-221.

25. Schrag D, Kuntz KM, Garber JE, Weeks JC. Life expectancy gains from cancer prevention strategies for women with breast cancer and BRCA1 or BRCA2 mutations. JAMA 2000; 283: 617-624.

26. Grann VR, Jacobson JS, Thomason D, Hershman D, Heitjan DF, Neugut Al. Effect of prevention strategies on survival and quality-adjusted survival of women with BRCA1/2 mutations: an updated decision analysis. J Clin Oncol 2002; 20: 2520-2529. 